\title{
A NOTE ON THE CONVOLUTION OF PROBABILITY MEASURES
}

\author{
ERROL CABY
}

\begin{abstract}
Simple proofs of the continuity of the convolution of measures for certain topologies are presented. In addition, results on the compactness of probability measures and on infinite convolutions of probability measures are obtained.
\end{abstract}

1. Introduction. In this paper we study the behavior of uniform measures under convolution, this being one of the prime reasons for their invention. The methods used also yield both new results and simple proofs of existing results for the more familiar classes of tight and $\tau$-smooth measures.

$\S 2$ is an exposition of some of the basic properties of uniform measures. In $\S 3$ we prove the continuity of convolution on the class of uniform measures and obtain some results on compactness. Among the byproducts are a simple proof of the continuity of convolution on the class of $\tau$-smooth measures and a new result on the relative compactness of tight measures. In $\S 4$ convergence principles are obtained for uniform measures and $\tau$-smooth measures.

2. Preliminaries. Let $(\mathcal{X}, \mathcal{U})$ be a uniform space; the class of uniform measures on $(\mathcal{X}, \mathcal{U})$ is defined as follows. Let $\Delta(x, \mathcal{U})$ denote the space of all bounded uniformly continuous real-valued functions on $(\mathcal{X}, \mathcal{U})$ and let $\Delta(\mathcal{X}, \mathcal{U})$ be equipped with the supremum norm. A subset of $\Delta(X, \mathcal{U})$ will be called a U.E.B. set if it is uniformly equicontinuous and bounded. Let $\Delta^{*}(\mathcal{X}, \mathcal{U})$ be the dual of $\Delta(\mathcal{X}, \mathcal{Y})$ and $M_{s}$ the space of measures with finite support on $\chi$. The class of uniform measures on $(\mathcal{X}, \mathcal{U})$, denoted by $M_{u}(\mathcal{X}, \mathcal{U})$, is defined as the closure of $M_{s}$ in $\Delta^{*}(\mathcal{X}, \mathcal{U})$ for the topology of uniform convergence on the U.E.B. subsets of $\Delta(X, U)$.

The following are some of the properties of uniform measures. These results can be found in $[\mathbf{1}, \mathbf{6}, \mathbf{7}$, and $\mathbf{1 0}]$.

PROPOSITION 2.1. If $(\mathcal{X}, \mathcal{U})$ is a complete metric space, then $M_{u}(\mathcal{X}, \mathcal{U})$ is identical with $M_{t}(\mathcal{X})$, the class of bounded tight measures on $\mathcal{X}$.

DEFINITION. Let $D$ be a family of pseudometrics on a set $\mathcal{X}$. We shall say that $D$ is directed if $\rho_{1}, \rho_{2} \in D$ implies the existence of a $\rho_{3} \in D$ such that for all $x, y \in X$

$$
\max \left\{\rho_{1}(x, y), \rho_{2}(x, y)\right\} \leq \rho_{3}(x, y) .
$$

Let $\rho$ be a pseudometric on a set $\mathcal{X}$; we shall let $\chi_{\rho}$ denote the metric quotient of the pseudometric space $(\mathcal{X}, \rho)$ and $\hat{\chi}_{\rho}$ denote the completion of $\chi_{\rho}$. In addtion we shall let $\pi_{\rho}$ denote the quotient map of $\mathcal{X}$ into $\hat{\chi}_{\rho}$.

Received by the editors December 20, 1985.

1980 Mathematics Subject Classification (1985 Revision). Primary 28C10, 60B11, 60B15.

Key words and phrases. Uniform space, uniform measures, tight measures, $\tau$-smooth measures. 
Proposition 2.2. Let $(\mathcal{X}, \mathcal{U})$ be a uniform space and let $D$ be a directed family of pseudometrics which generate $\mathcal{U}$. Let $\mu \in \Delta^{*}(\mathcal{X}, \mathcal{U})$. Then $\mu$ is a uniform measure if and only if $\pi_{\rho} \mu \in M_{t}\left(\hat{X}_{\rho}\right)$ for each $\rho \in D$.

REMARK. From Proposition 2.2 we can deduce that $M_{\tau} \subset M_{u}$, where $M_{\tau}$ is the class of $\tau$-smooth linear functionals on $\Delta(\mathcal{X}, \mathcal{U})$. It should be noted that there are spaces such that $M_{\tau}$ is strictly contained in $M_{u}$ (see [10]).

We shall now state some results on convergence and compactness in $M_{u}$.

PROPOSITION 2.3. On $M_{u}^{+}$the topology of uniform convergence on the U.E.B. subsets of $\Delta(X, \mathcal{U})$ coincides with the topology of weak convergence.

Proposition 2.4. Let $A$ be a subset of $M_{u}^{+}$. Then $A$ is weakly relatively compact in $M_{u}$ if and only if $\pi_{\rho} A$ is tight on $\hat{X}_{\rho}$ for each $\rho \in D$, where $D$ is a directed family of pseudometrics which generates the uniformity on $\mathcal{X}$.

Let us proceed to the case where $\mathcal{X}$ has the additional structure of a group. In particular let us assume that $\mathcal{X}$ is a Hausdorff topological abelian group. Let $\mathcal{U}$ be the associated uniformity. For $f \in \Delta(\mathcal{X}, \mathcal{U})$ and $x \in \mathcal{X}$, let $\left(\tau_{x} f\right)(y)=f(x+y)$. If $\nu \in \Delta^{*}(\mathcal{X}, \mathcal{U})$, then $\nu\left(\tau_{x} f\right)$ is a bounded uniformly continuous function on $(\mathcal{X}, \mathcal{U})$ and therefore if $\mu$ is another functional in $\Delta^{*}(\chi, U)$ we can form the expression $\mu\left[\nu\left(\tau_{x} f\right)\right]$. This expression defines a continuous linear functional on $\Delta(\mathcal{X}, \mathcal{U})$ which is called the convolution of $\mu$ and $\nu$ (see [8, p. 262]); that is $(\mu * \nu)(f)=\mu\left[\nu\left(\tau_{x} f\right)\right]$. This convolution operation, however, need not be commutative when considered on the whole space $\Delta^{*}$. This drawback, among other reasons, led LeCam in [9] to invent uniform measures, a large class of linear functionals on which the above convolution operation is commutative.

LEMMA 2.5. Let $\chi$ be a topological abelian group and let $\rho$ be a uniformly continuous pseudometric on $\chi$ which is compatible with the group operation. Let $\mu$ and $\nu$ be linear functionals in $\Delta^{*}(\mathcal{X}, \mathcal{U})$. Then $\pi_{\rho}(\mu * \nu)=\left(\pi_{\rho} \mu\right) *\left(\pi_{\rho} \nu\right)$.

Proof. Let $f \in \Delta\left(\hat{X}_{\rho}, \rho\right)$. Then

$$
\left[\pi_{\rho}(\mu * \nu)\right](f)=(\mu * \nu)\left(f \circ \pi_{\rho}\right)=\mu\left[\nu\left(\tau_{x}\left(f \circ \pi_{\rho}\right)\right)\right] \quad \text { where } x \in X .
$$

But $\pi_{\rho} \nu\left(\tau_{\pi_{\rho} x} f\right)=\nu\left(\tau_{x}\left(f \circ \pi_{\rho}\right)\right)$. Therefore

$$
\begin{aligned}
{\left[\pi_{\rho}(\mu * \nu)\right](f) } & =\mu\left[\pi_{\rho} \nu\left(\tau_{\pi_{\rho} x} f\right)\right] \\
& =\left(\pi_{\rho} \mu\right)\left[\left(\pi_{\rho} \nu\right)\left(\tau_{y} f\right)\right] \quad \text { where } y \in \hat{\mathcal{X}}_{\rho} \\
& =\left[\left(\pi_{\rho} \mu\right) *\left(\pi_{\rho} \nu\right)\right](f) .
\end{aligned}
$$

LEMMA 2.6. Let $\chi$ and $\rho$ be as in Lemma 2.5. Let $\mu$ be a linear functional in $\Delta^{*}(\mathcal{X}, \mathcal{U})$. Then $\pi_{\rho} \bar{\mu}=\overline{\pi_{\rho} \mu}$, where $\bar{\mu}(f)=\mu(\bar{f})$ and $\bar{f}(x)=f(-x)$.

PROOF.

$$
\left(\pi_{\rho} \bar{\mu}\right)(f)=\bar{\mu}\left(f \circ \pi_{\rho}\right)=\mu \overline{\left(f \circ \pi_{\rho}\right)}, \quad \text { where } f \in \Delta\left(\hat{\chi}_{\rho}, \rho\right) .
$$

But

$$
\overline{f \circ \pi_{\rho}}=\bar{f} \circ \pi_{\rho}, \quad\left(\pi_{\rho} \bar{\mu}\right)(f)=\mu\left(\bar{f} \circ \pi_{\rho}\right)=\left(\pi_{\rho} \mu\right)(\bar{f})=\overline{\left(\pi_{\rho} \mu\right)}(f) .
$$

3. The convolution operator. We shall now obtain some results on the behavior of uniform measures under convolution. In this section $\mathcal{X}$ will be a Hausdorff topological abelian group and $\mathcal{U}$ the associated uniformity. 
LEMMA 3.1. Let $\mu_{1}, \mu_{2}, \nu_{1}$, and $\nu_{2}$ be uniform measures. If $\rho$ is a uniformly continuous pseudometric on $\chi$ and if $S_{\rho}=\{f:|f(x)-f(y)| \leq \rho(x, y)$ for all $x, y \in \mathcal{X},\|f\| \leq 1\}$, then

$$
\begin{aligned}
\sup _{f \in S_{\rho}}\left|\left(\mu_{1} * \nu_{1}\right)(f)-\left(\mu_{2} * \nu_{2}\right)(f)\right| & \\
& \leq \sup _{f \in\left\|\nu_{1}\right\| S_{\rho}}\left|\mu_{1}(f)-\mu_{2}(f)\right|+\sup _{f \in\left\|\mu_{2}\right\| S_{\rho}}\left|\nu_{1}(f)-\nu_{2}(f)\right| .
\end{aligned}
$$

Proof. Let $f \in S_{\rho}$. Then

$$
\begin{aligned}
\mid\left(\mu_{1} * \nu_{1}\right)(f)- & \left(\mu_{2} * \nu_{2}\right)(f) \mid \\
& \leq\left|\left(\mu_{1} * \nu_{1}\right)(f)-\left(\mu_{2} * \nu_{1}\right)(f)\right|+\left|\left(\mu_{2} * \nu_{1}\right)(f)-\left(\mu_{2} * \nu_{2}\right)(f)\right| \\
& =\left|\mu_{1}\left[\nu_{1}\left(\tau_{x} f\right)\right]-\mu_{2}\left[\nu_{1}\left(\tau_{x} f\right)\right]\right|+\left|\nu_{1}\left[\mu_{2}\left(\tau_{x} f\right)\right]-\nu_{2}\left[\mu_{2}\left(\tau_{x} f\right)\right]\right| .
\end{aligned}
$$

However $\nu_{1}\left(\tau_{x} f\right)$ belongs to $\left\|\nu_{1}\right\| S_{\rho}$ and $\mu_{2}\left(\tau_{x} f\right)$ belongs to $\left\|\mu_{2}\right\| S_{\rho}$, so

$$
\left|\left(\mu_{1} * \nu_{1}\right)(f)-\left(\mu_{2} * \nu_{2}\right)(f)\right| \leq \sup _{f \in\left\|\nu_{1}\right\| S_{\rho}}\left|\mu_{1}(f)-\mu_{2}(f)\right|+\sup _{f \in\left\|\mu_{2}\right\| S_{\rho}}\left|\nu_{1}(f)-\nu_{2}(f)\right| \text {. }
$$

The lemma now follows.

From this lemma we can obtain the following result.

THEOREM 3.2. Let $\left\{\mu_{\alpha}\right\}$ and $\left\{\nu_{\alpha}\right\}$ be nets in $M_{u}$ and let $\left\{\nu_{\alpha}\right\}$ belong to a ball in $M_{u}$. Suppose $\left\{\mu_{\alpha}\right\}$ and $\left\{\nu_{\alpha}\right\}$ converge to the uniform measures $\mu$ and $\nu$, respectively, for the topology of uniform convergence on the U.E.B. subsets of $\Delta(\mathcal{X}, \mathcal{U})$. Then $\mu_{\alpha} * \nu_{\alpha}$ converges to $\mu * \nu$ for the topology of uniform convergence on the U.E.B. subsets of $\Delta(\mathcal{X}, \mathcal{U})$.

REMARK. Note that the measures in the above theorem need not be positive.

Recall that on $M_{u}^{+}$the topology of weak convergence coincides with the topology of uniform convergence on the U.E.B. subsets of $\Delta(\mathcal{X}, \mathcal{U})$. Thus we have proved the following result.

PROPOSITION 3.3. Let $\left\{\mu_{\alpha}\right\}$ and $\left\{\nu_{\alpha}\right\}$ be nets of positive uniform measures. If $\mu_{\alpha}$ and $\nu_{\alpha}$ converge weakly to the uniform measures $\mu$ and $\nu$ respectively, then $\mu_{\alpha} * \nu_{\alpha}$ converges weakly to $\mu * \nu$.

An immediate consequence is the following result which can be found in [4 and 5].

COROLLARY. Let $\left\{\mu_{\alpha}\right\}$ and $\left\{\nu_{\alpha}\right\}$ be nets of positive $\tau$-smooth measures on $\mathcal{X}$. If $\mu_{\alpha}$ and $\nu_{\alpha}$ converge weakly to the $\tau$-smooth measures $\mu$ and $\nu$ respectively, then $\mu_{\alpha} * \nu_{\alpha}$ converges to $\mu * \nu$.

Let us now look at compactness in $M_{u}^{+}$.

DEFinition. A linear functional $\mu$ in $\Delta^{*}(\mathcal{X}, \mathcal{U})$ is called a mean if $\mu \geq 0$ (that is, $\mu(f) \geq 0$ for $f \geq 0$ ) and $\mu(1)=1$. If a linear functional is both a mean and a uniform measure it is called a uniform mean.

PROPOSITION 3.4. Let $\left\{\lambda_{\alpha}\right\},\left\{\mu_{\alpha}\right\}$, and $\left\{\nu_{\alpha}\right\}$ be nets of uniform means on $\chi$ such that $\lambda_{\alpha}=\mu_{\alpha} * \nu_{\alpha}$ for each $\alpha$. If the nets $\left\{\lambda_{\alpha}\right\}$ and $\left\{\mu_{\alpha}\right\}$ are weakly relatively compact, then so is the net $\left\{\nu_{\alpha}\right\}$.

PROOF. Let $D$ be a directed family of pseudometrics such that each pseudometric in $D$ is compatible with the group operation and such that $D$ generates the 
topology on $\mathcal{X}$. Let $\rho \in D$. Then $\pi_{\rho} \lambda_{\alpha}, \pi_{\rho} \mu_{\alpha}$, and $\pi_{\rho} \nu_{\alpha}$ are tight probability measures on $\hat{X}_{\rho}$ and $\pi_{\rho} \lambda_{\alpha}=\left(\pi_{\rho} \mu_{\alpha}\right) *\left(\pi_{\rho} \nu_{\alpha}\right)$. Furthermore, since $\left\{\lambda_{\alpha}\right\}$ and $\left\{\mu_{\alpha}\right\}$ are weakly relatively compact in $M_{u}^{+},\left\{\pi_{\rho} \lambda_{\alpha}\right\}$ and $\left\{\pi_{\rho} \mu_{\alpha}\right\}$ are tight on $\hat{X}_{\rho}$. But if $\left\{\pi_{\rho} \lambda_{\alpha}\right\}$ and $\left\{\pi_{\rho} \mu_{\alpha}\right\}$ are tight on $\hat{X}_{\rho}$, then $\left\{\pi_{\rho} \nu_{\alpha}\right\}$ is also tight on $\hat{X}_{\rho}$ (see [11, Theorem 2.1, p. 58]). Thus, for each $\rho \in D,\left\{\pi_{\rho} \nu_{\alpha}\right\}$ is tight on $\hat{X}_{\rho}$ and so $\left\{\nu_{\alpha}\right\}$ is weakly relatively compact in $M_{u}^{+}$.

COROLLARY. Let $\left\{\lambda_{\alpha}\right\},\left\{\mu_{\alpha}\right\}$, and $\left\{\nu_{\alpha}\right\}$ be nets of tight probability measures on $\mathcal{X}$ such that $\lambda_{\alpha}=\mu_{\alpha} * \nu_{\alpha}$ for each $\alpha$. If the nets $\left\{\lambda_{\alpha}\right\}$ and $\left\{\mu_{\alpha}\right\}$ are weakly relatively compact in $M_{t}(\mathcal{X})$, then $\left\{\nu_{\alpha}\right\}$ is also weakly relatively compact in $M_{t}(\mathcal{X})$.

First we need the following lemma.

LEMMA 3.5. Let $\lambda$ and $\mu$ be tight probability measures on $\mathcal{X}$ and let $\nu$ be a uniform mean on $\chi$. If $\lambda=\mu * \nu$, then $\nu$ is also tight.

ProOF. The proof is similar to Lemma 3.1 of [2]. Also see [5].

PROOF OF THE COROLlaRY. From Proposition 3.4 it follows that $\left\{\nu_{\alpha}\right\}$ is weakly relatively compact in $M_{u}$. Let $\nu$ be a limit point of $\left\{\nu_{\alpha}\right\}$. Then $\nu \in M_{u}$ and there exist limit points $\lambda$ and $\mu$ of $\left\{\lambda_{\alpha}\right\}$ and $\left\{\mu_{\alpha}\right\}$ respectively, which satisfy $\lambda=\mu * \nu$ by Proposition 3.3. But $\lambda$ and $\mu$ are tight, therefore, by Lemma 3.5, $\nu$ is also tight. Thus $\left\{\nu_{\alpha}\right\}$ is weakly relatively compact in $M_{t}$.

REMARK. It can be easily shown that if $\left\{\lambda_{\alpha}\right\}$ and $\left\{\mu_{\alpha}\right\}$ are tight, then $\left\{\nu_{\alpha}\right\}$ is also tight. The above corollary takes this a step further and shows that this result is still true when tightness is replaced by weak relative compactness. It should be noted that for a general topological space tightness and relative weak compactness are not equivalent concepts for subsets of $M_{t}$.

4. Infinite convolutions of uniform measures. Let us turn to linear spaces. For these spaces we shall obtain results for uniform measures and $\tau$-smooth measures which are along the lines of Kloss's convergence principle. This principle has been proved for tight measures on topological groups (see $[\mathbf{3}, \mathbf{5}$, and $\mathbf{1 3}]$ ).

First we need the following result which can be found in [12].

THEOREM 4.1. Let $\mathcal{X}$ be a topological group and let $\mu$ and $\nu$ be tight probability measures on $\chi$. If $\mu * \nu=\mu$, then the closed subgroup generated by the support of $\nu$ is necessarily compact.

The following result is actually a refinement, which is possible for linear spaces, of Proposition 5 of $[\mathbf{1 3}]$ or Theorem 2 of $[\mathbf{5}]$.

LEMMA 4.2. Let $\mathcal{X}$ be a metrizable linear space which need not be locally convex. Let $\left\{\mu_{n}\right\}$ be a sequence of symmetric tight probability measures on $\chi$ and let $\nu_{\eta}=$ $\mu_{1} * \mu_{2} * \mu_{3} * \cdots * \mu_{n}$. If $\nu$ and $\nu^{\prime}$ are any two tight limit points of $\left\{\nu_{n}\right\}$, then $\nu=\nu^{\prime}$.

PROOF OF LEMMA 4.2. Let $\nu$ and $\nu^{\prime}$ be limit points of $\left\{\nu_{n}\right\}$. Since $\mathcal{X}$ is metrizable we can find two subsequences of $\left\{\nu_{n}\right\},\left\{\nu_{n_{m}}\right\}$, and $\left\{\nu_{n_{p}}\right\}$, such that $\nu_{n_{m}}$ converges weakly to $\nu$ and $\nu_{n_{p}}$ converges weakly to $\nu^{\prime}$.

Now $\nu_{n_{p}}=\nu_{n_{m}} * \nu_{n_{m} n_{p}}$ when $n_{m}<n_{p}$ and where $\nu_{n_{m} n_{p}}=\mu_{n_{m}+1} * \mu_{n_{m}+2} * \cdots *$ $\mu_{n_{p}} .\left\{\nu_{n_{p}}\right\}$ and $\left\{\nu_{n_{m}}\right\}$ are weakly relatively compact in $M_{t}$ and so, by the corollary to Proposition 3.4, $\left\{\nu_{n_{m} n_{p}}\right\}$ is also weakly relatively compact in $M_{t}$. Therefore, 
$\nu^{\prime}=\nu * \lambda$, where $\lambda$ is a limit point of $\nu_{n_{m} n_{p}}$. Since each $\mu_{n}$ is symmetric, it follows that $\nu^{\prime}, \nu$, and $\lambda$ are also symmetric. Similarly $\nu=\nu^{\prime} * \lambda^{\prime}$ where $\lambda^{\prime}$ is symmetric. It follows that $\nu=\nu *\left(\lambda * \lambda^{\prime}\right)$ and $\nu^{\prime}=\nu^{\prime} *\left(\lambda^{\prime} * \lambda\right)$. By Theorem 4.1 the support of $\lambda * \lambda^{\prime}, S_{\lambda * \lambda^{\prime}}$, is contained in a compact subspace of $\mathcal{X}$. However $\mathcal{X}$ is a Hausdorff topological vector space and therefore $\{0\}$ is the only compact subspace of $\chi$. Hence $S_{\lambda * \lambda^{\prime}}=\{0\}$. But $S_{\lambda * \lambda^{\prime}}=$ closure of $S_{\lambda}+S_{\lambda^{\prime}}$ and so $S_{\lambda}+S_{\lambda^{\prime}}=\{0\}$. However, as noted above, $\lambda$ and $\lambda^{\prime}$ are symmetric which implies that $S_{\lambda}=S_{\lambda^{\prime}}=\{0\}$. Therefore $\nu=\nu^{\prime}$.

THEOREM 4.3. Let $\mathcal{X}$ be a Hausdorff topological vector space which need not be locally convex. Let $\left\{\mu_{n}\right\}$ be a sequence of symmetric uniform means on $\mathcal{X}$ and let $\nu_{n}=\mu_{1} * \mu_{2} * \cdots * \mu_{n}$. If $\nu$ and $\nu^{\prime}$, two uniform means, are limit points of the sequence $\left\{\nu_{n}\right\}$, then $\nu=\nu^{\prime}$.

Furthermore if $\left\{\nu_{n}\right\}$ is weakly relatively compact in $M_{u}$, then $\left\{\nu_{n}\right\}$ converges.

PROOF. We shall prove this theorem by mapping $\chi$ into the metric spaces $\hat{X}_{\rho}$, $\rho \in D$, where $D$ is as before. Let $\rho \in D$. Then $\pi_{\rho} \mu_{k}$ and $\pi_{\rho} \nu_{k}, k=1,2,3, \ldots$, are symmetric tight probability measures on $\hat{\chi}_{\rho}$. Also $\pi_{\rho} \nu_{n}=\left(\pi_{\rho} \mu_{1}\right) *\left(\pi_{\rho} \mu_{2}\right) * \cdots *$ $\left(\pi_{\rho} \mu_{n}\right)$ by Lemma 2.5. Suppose that $\nu$ and $\nu^{\prime}$, two uniform means, are limit points of $\left\{\nu_{n}\right\}$. Then $\pi_{\rho} \nu$ and $\pi_{\rho} \nu^{\prime}$ are tight limit points of $\left\{\pi_{\rho} \nu_{n}\right\}$. By Lemma 4.2 it follows that $\pi_{\rho} \nu=\pi_{\rho} \nu^{\prime}$ and therefore $\nu=\nu^{\prime}$.

Suppose $\left\{\nu_{n}\right\}$ is weakly relatively compact in $M_{u}$. Then the limit points of $\left\{\nu_{n}\right\}$ are identical and therefore $\left\{\nu_{n}\right\}$ converges.

COROLLARY. Let $\mathcal{X}$ be a Hausdorff topological vector space which need not be locally convex. Let $\left\{\mu_{n}\right\}$ be a sequence of symmetric $\tau$-smooth probability measures on $\mathcal{X}$ and let $\nu_{n}=\mu_{1} * \mu_{2} * \cdots * \mu_{n}$. If $\nu$ and $\nu^{\prime}$ are two $\tau$-smooth limit points of $\left\{\nu_{n}\right\}$, then $\nu=\nu^{\prime}$.

Furthermore if $\left\{\nu_{n}\right\}$ is weakly relatively compact in $M_{\tau}$, then $\left\{\nu_{n}\right\}$ converges.

\section{REFERENCES}

1. E. Caby, The weak convergence of uniform measures, J. Multivariate Anal. 9 (1979), 130137.

2. _ On the extension of uniform measures, Proc. Amer. Math. Soc. 89 (1983), 433-439.

3. I. Csiszar, On infinite products of random elements and infinite convolutions of probability distributions on locally compact groups, Z. Wahrsch. Verw. Gebiete 5 (1965), 279-295.

4. __ Some problems concerning measures on topological spaces and convolutions of measures on topological groups, Les Probabilités sur les Structures Algébriques, C.N.R.S., Paris, 1970, pp. 75-96.

5. __ On the weak ${ }^{*}$ convergence of convolution in a convolution algebra over an arbitrary group, Studia Sci. Math. Hungar. 6 (1971), 27-40.

6. Z. Frolik, Mesures uniformes, C. R. Acad. Sci. Paris Ser. A 277 (1973), 105-108.

7. ___, Représentation de Riesz des mesures uniformes, C. R. Acad. Sci. Paris Ser. A 277 (1973), 163-166.

8. E. Hewitt and K. Ross, Abstract harmonic analysis. Vol. 1, Springer-Verlag, New York, 1979.

9. L. Le Cam, Remarques sur le théorème limite central dans les espaces localement convexes, Les Probabilités sur les Structures Algébriques, C.N.R.S., Paris, 1970, pp. 233-249.

10. _ Note on a certain class of measures, Unpublished.

11. K. Parthasarathy, Probability measures on metric spaces, Academic Press, New York, 1967. 
12. A. Tortrat, Lois de probabilite sur un espace topologique completement régulier et produits infinis à termes indépendant dans un groupe topologique, Ann. Inst. H. Poincaré 1 (1965), 217-237.

13. L_L Lois tendues et convolutions démombrables dans un groupe topologique $\chi$, Ann. Inst. H. Poincaré 2 (1966), 279-298.

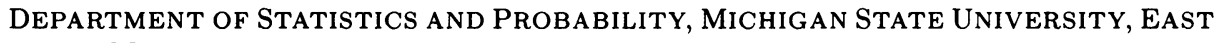
LANSING, MICHIGAN 48824

Current address: AT \& T, Room 7148L1, 295 North Maple Avevenue, Basking Ridge, New Jersey 07920 